Revue d'histoire de l'Amérique française

REVUE D.HISTOIRE DE L'AMÉRIQUE FRANÇAISE

\title{
Les premiers journaux du district de Saint-François (1823-1845)
}

\section{Jean-Pierre Kesteman}

Volume 31, numéro 2, septembre 1977

URI : https://id.erudit.org/iderudit/303610ar

DOI : https://doi.org/10.7202/303610ar

Aller au sommaire du numéro

Éditeur(s)

Institut d'histoire de l'Amérique française

ISSN

0035-2357 (imprimé)

1492-1383 (numérique)

Découvrir la revue

Citer cette note

Kesteman, J.-P. (1977). Les premiers journaux du district de Saint-François (1823-1845). Revue d'histoire de l'Amérique française, 31(2), 239-253.

https://doi.org/10.7202/303610ar d'utilisation que vous pouvez consulter en ligne.

https://apropos.erudit.org/fr/usagers/politique-dutilisation/ 


\title{
$-\mathrm{II}-$ \\ LES PREMIERS JOURNAUX DU DISTRICT DE SAINT-FRANÇOIS (1823-1845)
}

\author{
JEAN-PIERRE KESTEMAN \\ Département d'bistoire \\ Université de Sherbrooke
}

L'attrait récent pour l'histoire de la presse périodique au Québec n'a pu encore dissiper les zones d'ombre qui recouvrent l'activité journalistique dans les régions éloignées de Québec ou de Montréal. Sur les Cantons de l'Est, par exemple, nos connaissances restent encore très fragmentaires ${ }^{1}$. Dans les pages qui suivent, on trouvera une étude préliminaire des journaux publiés entre 1823 et 1845 dans le District de Saint-François. Préciser l'histoire de ces publications, mesurer leur rôle dans les tensions que connurent les comtés de Sherbrooke et de Stanstead dans les années précédant l'insurrection de 1837-38, expliciter les causes de leurs déboires ou de leur disparition, tels sont les fils conducteurs qui formeront la trame de cet article.

À part le British Colonist de Stanstead des années 1823 à 1826 , tous les journaux étudiés dans cet article ne subsistent plus qu'à l'état de numéros rares et isolés. Pour retrouver une trace plus régulière des journaux du District de Saint-François, il a fallu dépouiller des journaux anglophones de Montréal comme le Montreal Gazette (1828-1845) ou le Vindicator (1828-1837) ou une feuille des Cantons

1 À part les notices contenues dans A. Beaulieu et J. Hamelin, Les Journaux du Québec de 1764 à 1964 (Québec, P.U.L., 1965; cité désormais Journaux) et, des mêmes auteurs, La Presse québécoise des origines à nos jours I: 1764-1859 (Québec, P.U.L., 1973; cité dorénavant: La Presse...), on trouvera quelques éléments d'investigation chez Maurice O'Bready, Les journaux publiés dans les Cantons de l'Est depuis 150 ans, conférence au poste CHLT (Sherbrooke) (11 avril 1965), texte ronéotypé, 1965,10 p. et chez Louis C. O'Neill, «Des recherches n'ont pu permettre de déterminer le nombre exact de journaux qu'il y a eu à Sherbrooke», La Tribune (16 décembre 1961): 19. Voir aussi: La Tribune (31 juillet 1937): 4. 
de l'Est comme le Missiskoui Standard qui, selon un usage courant à l'époque, citent de larges et fréquents extraits de la presse de Sherbrooke ou de Stanstead. L'une ou l'autre vérification, pratiquée à partir d'un original local, nous laisse croire que ces citations étaient habituellement fidèles et peuvent donc constituer une intéressante base documentaire.

\section{Le British Colonist and St. Francis Gazette de Stanstead (1823-1834)}

Le premier journal publié dans les Cantons de l'Est vit le jour l'année de la création du District judiciaire de Saint-François ${ }^{2}$. En 1823, en effet, paraissait le numéro initial du British Colonist and St. Francis Gazette ${ }^{3}$. Bien que le village de Sherbrooke ait été choisi comme chef-lieu du district, c'est Stanstead qui fut le berceau de la presse de la région. À l'époque, ce gros bourg marchand demeurait la porte d'entrée des Cantons de l'Est qui, mal pourvus en chemins vers Québec ou Montréal, se greffaient au système de routes, de diligences et de courrier de la Nouvelle-Angleterre. Sa population nombreuse et cultivée, son activité commerciale à la frontière des ÉtatsUnis constituaient pour un journaliste une clientèle attirante ${ }^{4}$.

Le maitre d'œuvre de ce premier journal, propriétaire, rédacteur et imprimeur tout à la fois, Silas Horton Dickerson, était comme la plupart des habitants de la région de Stanstead, originaire des États-Unis ${ }^{5}$. Pendant plus de onze ans ${ }^{6}$, il rédigea son hebdomadaire

2 Antérieurement à cette date, deux journaux du Vermont, le Green Mountain Patriot, de Peacham et le North Star, de Danville, étaient distribués dans les townships de Stanstead, Barnston, Hatley et Compton, deux fois par mois par un service postal à cheval des États-Unis. cf., B. F. Hubbard, Forests and clearings. The history of Stanstead County (Montréal, 1874), 16.

3 A. Beaulieu et J. Hamelin, Journaux, 268; La Presse..., 46-47; JeanPierre Kesteman, "Le premier journal des Cantons de l'Est», La Tribune (27 juillet, 3 août 1974). Voir aussi la fiche signalétique no 1 en annexe. Le journal sera cité Colonist.

4 En 1827, Stanstead s'enorgueillit d'une soixantaine de maisons bordant une large avenue de 132 pieds, de ses commerces proches de la frontière ( on the line»), de sociétés bibliques et d'une bibliothèque. Voir Colonist (18 décembre 1823, 6 septembre 1827).

5 On trouvera une brève biographie de Dickerson dans H. J. Morgan, Sketches of celebrated Canadians (Québec, 1862), 413 et dans B. F. Hubbard, op. cit., 150. Né au New-Jersey (1799), il devint apprenti imprimeur à Kingston, Ont. (1813), puis à Montréal. Installé en 1823 à Stanstead, réfugié aux États-Unis de 1837 à 1840 , receveur des douanes (1853), puis maire de Stanstead (1857). Il mourut le 23 octobre 1857. affirmé.

6 Le Colonist vécut jusqu'en 1834 et non en 1832, comme il est généralement 
au milieu de difficultés sans nombre. Les embûches qui guettaient une entreprise de presse dans les Cantons de l'Est vers 1825, étaient d'abord matérielles: les souscripteurs étaient dispersés sur un vaste territoire, le système de distribution tributaire d'un réseau routier primitif, l'approvisionnement en papier et en encre parfois aléatoire ${ }^{7}$. De plus, le manque de numéraire obligeait le journaliste à accepter le paiement de ses abonnements en nature ${ }^{8}$. Ces contraintes affectèrent très tôt la rentabilité de l'hebdomadaire, pour lequel Dickerson réclama sans cesse les paiements en souffrance, en vain d'ailleurs la plupart du temps ${ }^{9}$.

À cette fragilité financière qui fut le lot des journalistes en terre de pionniers, s'ajouta, à partir de 1826, la lutte harassante que menèrent successivement le pouvoir judiciaire et la classe des marchands conservateurs du district contre les idées et les positions politiques du Colonist. À ses débuts pourtant, Dickerson avait énoncé de façon rassurante sa foi dans les institutions britanniques:

The Subscriber, therefore, begs to acquaint the Inhabitants of the Townships (...) that the BRITISH COLONIST will be conducted upon the strictest principles of Patriotism, and its columns shall always have for their object the general good of the Settlements.

Impressed as he is, with a sincere attachment to the Constitution of Great Britain, the Subscriber's political conduct shall be entirely influenced by the principles of that glorious Bulwark of the Peoples' Rights ${ }^{10}$.

Durant les premières années de parution, le Colonist s'intéressait fort peu aux événements ou aux problèmes des Cantons de l'Est; on y trouvait plutôt des chroniques de religion et de morale, des lettres de missionnaires méthodistes, des récits de voyage ou anecdotes curieuses en plus de la reproduction de nouvelles européennes ou américaines empruntées aux journaux de Boston et de New York. Seules les annonces des marchands de la région donnaient une note locale aux pages du Colonist. Cependant, à partir de 1826, l'hebdomadaire de Stanstead avait ouvert progressivement ses colonnes

7 Le journal fut parfois imprimé à format réduit ou même suspendu faute d'encre ou de papier. Cf. Colonist (3 juillet 1823, 2 septembre 1824).

8 Blé, blé d'inde, porc salé, beurre, fromage, sucre, suif et chiffons pour un montant de $\$ 2.50$ par an, si ces produits étaient livrés au bureau du journal. Cf. Colonist (1 ${ }^{\text {er }}$ mai 1823).

9 Colonist (3 juillet, 30 octobre 1823, 5 février 1824).

10 Colonist (1 $\mathrm{l}^{\mathrm{er}}$ mai 1823): 1. 
aux revendications qui ne cessaient d'être émises par des habitants des Townships auprès de la Chambre d'Assemblée du Bas-Canada: amélioration du système routier, création de bureaux d'enregistrement et de circonscriptions électorales, amendements au système judiciaire du district ${ }^{11}$.

Pour avoir inséré dans son journal des réflexions sur les procédés arbitraires du juge John Fletcher, du District de Saint-François, le rédacteur du Colonist fut arrêté plusieurs fois, condamné à l'amende et même à la prison pour outrage au tribunal ${ }^{12}$. Dickerson porta en 1829 cette affaire à la connaissance de la Chambre d'Assemblée du Bas-Canada, qui mena, pendant plusieurs sessions, enquête sur le comportement du juge Fletcher ${ }^{13}$. Ces démarches ne purent le réhabiliter ni le rembourser de l'argent perdu lors de ses démêlés avec la justice, mais lui valurent auprès des milieux progressistes la gloire d'un martyr pour la liberté de la presse ${ }^{14}$. Crut-il en tirer auprès de ses concitoyens un certain capital politique? Toujours est-il qu'il se présenta candidat lors de la première élection dans le nouveau comté

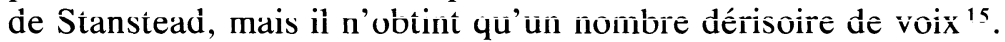

Désormais son engagement politique ira en se précisant ${ }^{16}$; déjà en 1826, le Colonist s'en prenait aux marchands de la région qu'il accusait de s'opposer au progrès pour mieux conserver leur emprise économique sur les cultivateurs:

Besides the Merchant who has a capital, has a greater chance to advance his property without good roads than he would have with them. He can procure his stores in the winter, and in the summer, when they can be obtained no other way, he can hold them at such prices as his avarice may dictate. ${ }^{17}$

11 Dickerson commença à partir du 27 juillet 1826 une chronique politique. Il publia bientôt les lettres de correspondants réguliers qui dissertaient du retard de la région sous des pseudonymes divers (Vindex, Philo Junius, Farmer...).

12 Maurice O'Bready, De Ktiné à Sherbrooke. Esquisse historique de Sherbrooke des origines à 1954 (Sherbrooke, 1973), 69-76; B. F. Hubbard, op. cit., 17-18.

13 Journaux de la Chambre d'Assemblée du Bas-Canada (1831-32): 434-436 et app. W; (1835-36): 603, 634-635 et app. EE.

14 Il est étonnant qu'aucune mention de cette affaire de liberté de presse ne soit faite dans le livre de Jean-Paul de Lagrave, Les journalistes-démocrates au Bas-Canada (1791-1840) (Montréal, 1975).

15 Vindicator (20 novembre 1829): 3.

16 Les numéros des années 1828-1834 ont presque tous disparu, mais les extraits qu'en ont tirés d'autres journaux, comme le Vindicator de Montréal, permettent de suivre l'évolution du Colonist.

17 Colonist (7 décembre 1826): 3 . 
Par la suite, son journal souligna les irrégularités qui auraient entouré l'élection de Samuel Brooks, marchand, dans le comté de Sherbrooke en $1829^{18}$, se montra favorable aux politiques de la majorité réformiste en Chambre ${ }^{19}$, couvrit avec enthousiasme les assemblées tenues dans le district en faveur des politiques progressistes, puis des 92 Résolutions ${ }^{20}$.

Dans le courant de 1834, en effet, le comté de Stanstead était ouvertement passé du côté réformiste et le député Marcus Child avait affirmé sans réticences sa foi radicale ${ }^{21}$. La popularité croissante des idées réformistes ${ }^{22}$ dans le District, propagées par le journal de Dickerson, ne pouvait qu'effrayer les milieux loyalistes; aussi, profitant du fait que le rédacteur du Colonist était criblé de dettes, contractées principalement durant ses procès avec le juge Fletcher, des marchands parvinrent à faire vendre son matériel par le shérif ${ }^{23}$. On peut dater cette saisie qui marque la fin du Colonist, de juin $1834^{24}$.

Bien que privé de son journal, Dickerson devint bientôt président de l'association réformiste de Stanstead et, à ce titre, fut jusqu'à l'insurrection de 1837, parmi les plus actifs défenseurs dans le District des idées de Papineau ${ }^{25}$.

\section{Le St. Francis Courier de Sherbrooke (1831-1835)}

Une certaine confusion persiste quant au nombre et à l'histoire des journaux publiés à Sherbrooke dans les années 1830. André Beaulieu et Jean Hamelin distinguaient dans un premier temps, le St. Francis Courier, réformiste (1831-1836), et le Farmer's and

18 Colonist cité par Vindicator (11 décembre 1829): 2.

19 Colonist cité par Vindicator (4 novembre 1831): 2.

20 Colonist cité par Vindicator (31 janvier 1834): 2; (18, 22 avril 1834).

21 Vindicator (2, 20 mai 1834).

22 Dans les Cantons de l'Est, les milieux hostiles aux tories se définissent comme «liberal people», «the Liberals», "Reformers». Voir p. ex. St. Francis Courier, cité par Vindicator (3 juin, 31 octobre 1834).

23 En octobre 1833 déjà, première vexation: son propriétaire le force à quitter les lieux loués pour son imprimerie dans les quinze jours (David Kenaston vs. Silas H. Dickerson, 12 octobre 1833, Archives judiciaires du District de Saint-François (citées désormais AJF), notaire Richardson, 683).

24 Le Vindicator en parle dans son édition du 27 juin. Voir aussi, Vindicator (16 septembre 1834) pour plus de détails.

25 Vindicator $(20,27$ janvier 1835). Il se réfugiera aux États-Unis de 1837 à 1840 (cf. Morgan, op. cit.). 
Mechanic's Journal, conservateur, organe de Joseph Walton, fondé en 1833, suspendu en 1837-1838 et rebaptisé Sherbrooke Gazette en $1840^{26}$. Par ailleurs, dans un ouvrage plus récent, les mêmes auteurs n'accordent à Sherbrooke qu'une seule entreprise de presse qui, de 1831 à 1840, aurait édité un hebdomadaire sous des titres divers: St. Francis Courier (1831-1836), Farmer's Advocate (1836-1837), Sherbrooke Gazette (1837-1839), Farmers' and Mechanics' Journal (1839), puis de nouveau Sherbrooke Gazette (1840 sq.) ${ }^{27}$. En fait, il est possible d'établir que, durant cette décennie, Sherbrooke connut, sous les titres précédents, au moins quatre journaux différents, parfois même concurrents. En plus de témoigner de l'importance prise par la presse dans le District de Saint-François, ces feuilles, aux options politiques variées, éclairent d'un jour nouveau le tissu idéologique des populations de la région pendant ces années d'agitation.

Le plus ancien journal de Sherbrooke est sans contredit le St. Francis Courier ${ }^{28}$. C'est, en effet, en août 1831 que les frères Calvin et Daniel Tolford arrivent des États-Unis avec leur matériel d'imprimenerie ${ }^{29}$. Bieñ que le Vindicator ait salué la nouvelle publication comme un champion de l'action progressiste dans le District ${ }^{30}$, le Courier semble, au début du moins, défendre des opinions modérées et nuancées, ce qui lui vaut d'être régulièrement cité par le Montreal Gazette ${ }^{31}$. Sous la plume de Calvin Tolford, on trouve en effet des articles pleins de réserves sur la réforme du Conseil législatif ${ }^{32}$, hostiles au suffrage universel ${ }^{33}$ ou favorables à une gestion autonome d'une taxe foncière pour l'amélioration des chemins dans les Cantons de l'Est:

All we want in the Townships is, laws adapted to the Townships

(...) French laws do very well for the French country but they

26 André Beaulieu et Jean Hamelin, Journaux, op. cit., 262.

27 André Beaulieu et Jean Hamelin, La Presse..., op. cit., 71-72.

28 Voir fiche signalétique no 2 en annexe. Cité désormais Courier.

29 Ils traversent Stanstead vers le 15 août. Cf. Montreal Gazette (1 $1^{\mathrm{er}}$ septembre 1831): 2 .

30 «We received a number of a new Journal named the St. Francis Courier published in Sherbrooke by the Messrs. Tolfords, which promises to be a very useful publication (...) It will be a valuable adjunct to Mr. Dickerson's Journal and will do much in diffusing those principles in its neighbourhood which the enlightened portion of mankind at the present day pronounces to be correct", in Vindicator (13 sept. 1831): 3 .

31 Qui lui emprunte des articles sur les récoltes et les faits-divers (26 novembre

1831, 1 er mars, 26 juillet, 25 octobre 1832,22 juin, 13 juillet 1833 , etc...).

32 Courier cité par Montreal Gazette (2 février 1832): 3.

33 Courier cité par Montreal Gazette (27 février 1832): 3. 
will never do for the Townships. We should have laws as distinct (...) from those of the French, as our people are distinct from the Canadians (...) strange that the people of the Townships should have so long submitted in silence to the present order of things. ${ }^{34}$

Ce n'est véritablement qu'à la fin de 1832 , lorsque se précisent les projets de la «British American Land Company», que le Courier prend ouvertement position contre l'établissement de cette entreprise dans le District. Changement d'attitude qui lui vaut la riposte immédiate des tories de Sherbrooke, dont les chefs de file, marchands du lieu, attendent les retombées du capital que la nouvelle compagnie projette d'investir. Aussi décident-ils de mettre au pas le journaliste récalcitrant; lors d'une réunion politique tenue à Sherbrooke en janvier 1833, Calvin Tolford se voit sommé d'imprimer dans son propre journal une résolution blâmant ses positions ${ }^{35}$.

Dès lors, le Courier s'attaque sans détours à ce qu'il considère comme «la conspiration des tories contre le peuple» ${ }^{36}$ et consacre de nombreux articles au progrès des idées réformistes dans le District $^{37}$; ce tournant politique lui vaut d'ailleurs de se faire accuser d'être financé par le parti de Papineau ${ }^{38}$ ! Le Courier de Sherbrooke rejoignait ainsi le Colonist de Stanstead pour constituer un monopole radical sur la presse des Cantons de l'Est. Pas pour longtemps, puisque nous avons vu que le journal de Stanstead fut vendu en juin 1834; racheté par les marchands, le matériel fut ramené à Sherbrooke et utilisé pour imprimer un nouveau journal, d'obédience conservatrice et dévoué aux intérêts de la «British American Land Company» le Farmers' Advocate ${ }^{39}$. En quelques jours, le Courier perdait un allié et trouvait un concurrent à Sherbrooke même.

34 Courier cité par Montreal Gazette (22 septembre 1832): 2.

35 "That the publication of certain articles which have appeared in the $S t$. Francis Courier in opposition to the establishment of the British American Land Company, has, in our opinion, militated against the interests of this Province in general, and this section in particular», texte cité par le Vindicator (16 septembre 1834): 3. Voir aussi Montreal Gazette (19 mars 1833): 1.

36 Courier cité par Vindicator (14 mai 1833): 2. («We sincerely believe there is a combination of political speculators in the Eastern Townships, to control the public voice and keep the people in ignorance as to their true wants and interests").

37 Le Vindicator (31 janvier 1834): 2, cite un long article du Courier qui éclaire bien ses nouvelles orientations.

38 Montreal Gazette (25 juillet 1833): 2; Vindicator (26 juillet 1833): 3 .

39 Vindicator (27 juin 1834): 2. 
Encouragés par cette première victoire, les marchands conservateurs se sentirent de taille à abattre le second journal réformiste du District. L'éditeur Calvin Tolford ayant malencontreusement contracté des dettes, ils parvinrent à rééditer l'exploit de Stanstead et firent saisir le matériel du Courier ${ }^{40}$. Il en allait de la diffusion des idées radicales dans la région et Tolford chercha des appuis à Montréal. Le 28 juillet 1834, Edmund B. O'Callaghan, rédacteur du Vindicator, se rendait à Sherbrooke pour donner en location à Tolford une presse et du matériel divers, à condition qu'il reprenne la publication du Courier ${ }^{41}$. Un mois plus tard, sans faire aucune allusion au rôle joué par son rédacteur, le Vindicator saluait la rentrée du journal de Sherbrooke ${ }^{42}$.

L'approche des élections, en octobre 1834, permit au Courier de donner toute sa mesure ${ }^{43}$. Son rival, le Farmers' Advocate le malmène avec d'autant plus de hargne ${ }^{44}$ que Calvin Tolford est bientôt choisi candidat «libéral» pour Sherbrooke ${ }^{45}$. Le scrutin, qui se déroula non sans intimidation de la part des marchands, vit la victoire de justesse des candidats conscrvateurs Gugy ct Moore aux dépens de Tolford et de son coéquipier Daniels ${ }^{46}$. Par contre dans le comté de Stanstead, les réformistes Child et Grannis écrasèrent leurs opposants; cette victoire fut célébrée dans l'enthousiasme quand, en janvier 1835, Louis-Joseph Papineau vint à Stanstead, entouré de neuf députés dont les montréalais Jacques Viger et Edmund O'Callaghan, ranimer les ardeurs radicales de ses partisans locaux ${ }^{47}$.

40 Vindicator (16 septembre 1834): 2. L'événement doit se situer en juillet 1834, un mois environ après la disparition du Colonist.

41 La transaction est faite au loyer annuel de $£ 12$. Tolford, qui a tout perdu dans la saisie de son journal, se voit offrir par O'Callaghan une théière et des casseroles (cf. E. B. O'Callaghan à C. W. Tolford, 28 juillet 1834, AJF, notaire Bureau, 255).

42 Vindicator (26 août 1834): 3.

43 L'édition du Courier du 2 octobre 1834, une des rares à avoir survécu, en est un bel exemple. On remarque qu'à cette date le journal n'a presque plus d'annonceurs!

44 L'existence pour la même semaine d'octobre 1834 d'un exemplaire de chacun des deux journaux prouve bien qu'il s'agit d'entreprises distinctes.

45 Vindicator (27 octobre 1834): 2; Courier cité par Vindicator (31 octobre 1834): 1 .

46 Courier cité par Vindicator (14 novembre 1834): 2 . Tolford obtint 155 voix contre 161 à Gugy et 168 à Moore, un agent de la «British American Land Company », et Daniels 151 .

47 On trouve une relation complète de cet événement dans le Vindicator (27 janvier 1835): 2-3. 
En 1835, l'activité politique marque le pas dans la région. Le Courier est encore cité à plusieurs reprises, sauf cependant pour ses opinions politiques ${ }^{48}$; soudainement, en décembre de la même année, il disparait ${ }^{49}$. Le Farmers' Advocate devient dès lors le seul journal du District. L'opinion est désormais contrôlée par les tories ${ }^{50}$.

\section{Le Farmers' Advocate de Sherbrooke (1834-1837)}

Le mercredi 23 juin 1834, sur les presses du défunt Colonist, sortit le premier numéro du second hebdomadaire de Sherbrooke, le Farmers' Advocate and Townships Gazette ${ }^{51}$. Propriété de MM. Walton et Gaylord ${ }^{52}$, il était rédigé par Joseph S. Walton, lui aussi d'origine américaine ${ }^{53}$. Bien que dans son premier éditorial, Walton ait peu précisé ses principes politiques, à part son indépendance ${ }^{54}$, on le voit rapidement défendre le point de vue conservateur et vanter la "British American Land Company ${ }^{55}$. Les journaux réformistes de l'époque le disaient à la solde de la Compagnie ${ }^{56}$ et cette affirmation n'est peut-être pas dénuée d'un certain fondement ${ }^{57}$. De toute façon,

48 Montreal Gazette (4 juin 1835): 3 ; Vindicator (8 septembre, 9 octobre 1835).

49 Sa rentabilité, faute d'annonceurs, devait être précaire. Le Missiskoui Standard (29 décembre 1835) félicite l'Advocate de Sherbrooke d'avoir vaincu «the revolutionary St. Francis Courier ", mort du retrait des subsides de Papineau!

so D'autant plus qu'à l'ouest du District circule un nouveau journal conservateur, le Missiskoui Standard (1835-1839) de Frelighsburg.

51 Montreal Gazette (26 juin 1834): 2; Vindicator (27 juin 1834): 2. Nous abrégeons désormais son titre en Advocate.

52 Asa Gaylord est un personnage mal connu; on le retrouve comme libraire et relieur à Stanstead de 1833 à 1840.

53 Joseph Soper Walton (1802-1875), né à Pencham (Vermont), en 1816, apprenti imprimeur chez son frère F. P. Walton à Montpelier (Vermont), en 1833, associé à Stanstead avec Asa Gaylord comme imprimeur et relieur; éditeur du Sherbrooke Gazette jusqu'en 1870.

54 Montreal Gazette (26 juin 1834): 2.

s5 Advocate cité par Montreal Gazette (8 juillet 1834): 2 ; (12 août 1834): 2.

56 «is ought rather be named The Land Company's Advocate» (Vindicator, 2 septembre 1834); Courier (2 octobre 1834).

57 La correspondance de Samuel Brooks, secrétaire de la British American Land Company et de John Moore, l'agent des terres à Lennoxville, reflète en tout cas un intérêt marqué pour l'Advocate. On y trouve, entre autres, une demande d'envoi d'un abonnement pour l'agence de Québec et pour le bureau principal à Londres; référence à l'expulsion prochaine de Tolford, rédacteur du Courier, de l'immeuble que la Compagnie lui louait, au profit de Walton qui s'y installerait; achat d'espace publicitaire par la Compagnie dans l'Advocate; informations confidentielles accordées à Walton en vue de publier des articles élogieux sur l'activité de la Compagnie (APC, British American Land Company Papers, Letter Book (1834-36): 894, 940, $950,954,958)$. 
le rédacteur de l'Advocate était membre actif de l'association conservatrice du comté, comme Tolford et Dickerson l'avaient été chez les réformistes, tant il est vrai que le journalisme de ces années agitées ne pouvait se concevoir sans quelque engagement politique.

Joseph Walton était un rédacteur de talent, à la plume agréable et variée ${ }^{58}$; son journal, bien informé, publia quelque temps une correspondance directe de Londres ${ }^{59}$. Malgré ses qualités, l'Advocate semble avoir eu des difficultés financières au printemps de $1836^{60}$, mais comme sa publication ne connut pas d'interruption cette année-là, on peut penser qu'il reçut des milieux conservateurs de Sherbrooke un encouragement quelconque; d'ailleurs, après une accalmie de plusieurs mois, les radicaux faisaient des projets pour établir un nouveau journal dans les Cantons de l'Est ${ }^{61}$. À la fin de l'année, l'Advocate, qui n'a cessé de vanter la libéralité et l'esprit d'entreprise de la «Compagnie des Terres» dans la région de Sherbrooke ${ }^{62}$, retrouve un regain d'ardeur politique; son rôle fut indéniable lors de l'élection partielle de Stanstead en 1837, quand le candidat réformiste Flias Lee se fit battre nettement par le conservateur Colby. Cette défaite révélait aux radicaux la faiblesse de leurs forces dans une région qu'ils contrôlaient jadis par leurs journaux; le Vindicator l'attribue autant à l'absence d'une feuille sympathique à leurs idées qu'à l'influence de l'Advocate, seul hebdomadaire circulant dans le comté et qui reproduisait les discours de Colby ${ }^{63}$.

Malgré ces succès d'estime, en juillet 1837, l'Advocate, qui défendait depuis trois ans l'évangile tory dans le District, cessait sa publication et était vendu par ses propriétaires à un avocat montréalais, Robert Armour, junior ${ }^{64}$.

58 Ainsi dans le numéro du 6 octobre 1834 trouve-t-on une pièce de théâtre mettant en scène trois personnages Marcus (Child, député de Stanstead), Calvin (Tolford) et Silas (Dickerson). Avec cynisme, les trois radicaux complotent pour tromper les «bons" électeurs du district, affaiblir la vente de l'Advocate, utiliser à des fins de corruption l'argent de Papineau et faire élire Child sous le masque d'un irréprochable ami de l'ordre et de la constitution.

59 Reprise par le Montreal Gazette (27, 29 janvier, 28 février 1835).

60 Vindicator (22 avril 1836): 3 («the printers wishing to engage in some other business»).

$61 \quad$ Vindicator (1 juillet 1836): 2.

62 Advocate cité par Missiskoui Standard (16 août, 18 octobre 1836).

63 Advocate cité par Missiskoui Standard (20 décembre 1836); Vindicator (20 janvier 1837): 3.

64 L'arrêt de la publication est signalé dans le Missiskoui Standard (8 août 1837): 3. La vente est faite au montant de £225. Cf. Joseph S. Walton à Robert Armour, Jr (15 septembre 1837) AJF, notaire Bureau, 867. 


\section{Les journaux radicaux de 1837-38}

Après la disparition du Colonist en 1834 et du Courier en 1835, les Cantons de l'Est demeuraient sans organe de presse réformiste. Le progrès des idées radicales, particulièrement dans la zone proche de la frontière peuplée de colons américains, devait cependant faire naitre de nouveaux journaux, souvent éphémères, parfois clandestins.

C'est d'abord dans le comté de Missisquoi, à l'extérieur du District, qu'apparut le Township Reformer de Stanbridge, dont la vie fut brève et la fin mouvementée ${ }^{65}$. Il avait été précédé du Missiskoui Post, publié au même endroit à partir de 1834, qui semble avoir également exprimé des opinions radicales ${ }^{66}$. À Sherbrooke, où la prudence s'imposait après l'échec du Courier, c'est sous le couvert de l'humour que s'expriment les sentiments anti-tories: dès juin 1837, circule dans les Cantons un petit journal satirique appelé le Skylark ${ }^{67}$, dont le titre s'orne de l'équerre et du compas franc-maçons. Quelques témoignages laissent penser qu'il s'en prenait avec plaisir à la «Compagnie des Terres» et au journal conservateur de Sherbrooke, l'Advocate, dont il salue la mort de façon ironique. On ignore si le Skylark lui survécut de longtemps.

Quant au Canadian Patriot ${ }^{68}$, il apparut durant l'hiver 1837-38, en pleine tourmente. Distribué à Stanstead bien qu'imprimé de l'autre côté de la frontière à Derby, au Vermont, il tentait de raffermir les sentiments insurrectionnels des radicaux du District en leur diffusant les résolutions d'appui aux patriotes des républicains américains et en leur prêchant la vigilance armée contre le «joug britannique» ${ }^{69}$.

65 On peut préciser les dates indiquées par A. Beaulieu et J. Hamelin (Journaux: 268; La Presse..., 99) de la façon suivante: premier numéro vers le 20 novembre 1836. Cf. Vindicator (25 novembre 1836): 3. Le journal dut disparaitre en août 1837 lorsque dans une émeute, sa presse et ses caractères furent brisés. Cf. Vindicator (11 août 1837): 2.

66 Ses débuts sont salués par le Montreal Gazette (20 décembre 1834): 2. Voir A. Beaulieu et J. Hamelin, La Presse..., 84.

67 Première mention dans le Vindicator (6 juin 1837): 1. Le numéro 5 (3 août 1837) est décrit par Louis C. O’Neill, «Des recherches n'ont pu permettre de déterminer le nombre exact de journaux qu'il y a eu à Sherbrooke», La Tribune (16 décembre 1961): 19. Voir fiche signalétique no 4. no 5 .

68 Voir A. Beaulieu et J. Hamelin, La Presse..., 98 et la fiche signalétique

69 On en trouvera de larges extraits chez Jules Martel, «Les troubles de 1837-38 dans la région de Sherbrooke», Revue de l'Université de Sherbrooke, V (1964): 39-58. Sur les événements, voir aussi: B. F. Hubbard, op. cit., 12-16. 
Son rédacteur Hiram-Francis Blanchard participa au raid manqué contre Stanstead en mars 1838 et fut arrêté en novembre. Le Patriot était bien un journal d'agitation et, comme tel, s'évanouit avec l'échec des activistes qui le dirigeaient.

\section{Le Sherbrooke Gazette (1837-38) et ses mutations (1839 sq.)}

Robert Armour jr, qui avait racheté les presses de l'Advocate, publia dès septembre 1837 un nouvel hebdomadaire qu'il coiffait du titre de Sherbrooke Gazette and Townships Advertiser ${ }^{70}$. S'affichant non sans ambiguité "constitutional reformer", il se déclare "en faveur du progrès dans le respect des autorités établies $»^{71}$. Entreprise de presse complètement nouvelle, dont les liens avec le Montreal Gazette sont assez probables ${ }^{72}$, le journal d'Armour se fera le porteparole des opinions loyalistes du District durant les troubles de 1837. $\grave{A}$ ce titre, on peut y trouver des comptes rendus des assemblées loyalistes à Compton et à Shipton ${ }^{73}$, des détails sur l'organisation des milices à Sherbrooke par le colone! Heriot ${ }^{74}$ ou sur le coup de main de Potton ${ }^{75}$. La période troublée que vivait alors le Bas-Canada fut fatale toutefois au nouvel éditeur, malgré ses opinions loyalistes. Problèmes d'approvisionnement en papier des États-Unis? Difficultés d'assurer la distribution régulière en période troublée dans une vaste région? Baisse de la clientèle dans un district qui comptait pas mal de radicaux? De nombreux motifs peuvent expliquer la suspension du Sherbrooke Gazette. C'est un fait qu'après mars 1838, le journal n'est plus cité et qu'après novembre de la même année, le Montreal Gazette couvre la région de Sherbrooke par un correspondant ${ }^{76}$. Il est probable que l'entreprise, en difficultés financières, dut revenir à son ancien propriétaire, Joseph Walton, l'ex-éditeur de l'Advocate, car celui-ci lança, en janvier 1839, le Farmer's and Mechanics'

70 Le premier numéro du 23 septembre 1837 fut salué par le Montreal Gazette (28 septembre 1837), le Vindicator (29 septembre 1837) et le Missiskoui Standard (3 octobre 1837). Voir la fiche signalétique no 6.

71 Sherbrooke Gazette (23 septembre 1837): 2.

72 Robert Armour junior est sans doute le fils de Robert Armour, rédacteuren-chef du Montreal Gazette de 1809 à 1845 et propriétaire de 1826 à 1844 (cf. A. Beaulieu et J. Hamelin, La Presse..., 6). Le Montreal Gazette (17 octobre 1837) fait de la publicité pour le journal de Sherbrooke.

73 Sherbrooke Gazette (28 décembre 1837): 2.

74 Montreal Gazette (7 décembre 1837): 1.

75 Montreal Gazette (13 mars 1838): 3.

76 Montreal Gazette (17, 20 novembre, 6, 13 décembre 1838). 
Journal ${ }^{77}$. Il ne fait aucun doute que ce journal changea simplement de titre à une date subséquente pour s'appeler Sherbrooke Gazette and Eastern Townships Advertiser, mais tout laisse croire que cette modification se produisit au début de 1843 et non de $1840^{78}$. L' hebdomadaire de Walton restera le seul journal dans le District jusqu'en 1845, année de fondation du Stanstead Journal et le seul à Sherbrooke jusqu'à l'apparition de l'éphémère St. Francis Telegraph en 1851. Ce quasi-monopole de l'information reflète bien l'état de la vie politique des Cantons de l'Est où dominent à l'époque les partisans de l'Union et les plus fidèles appuis aux thèses de Metcalfe, connus sous le nom de groupe "British Tory ${ }^{79}$. Les radicaux des années 1834-1838 étaient ou bien retirés aux États-Unis, ou bien réduits au silence par la pauvreté; le régime de l'Union donnait à la population anglophone des Cantons de l'Est toutes les espérances d'un développement politique et économique harmonieux; les débuts du système municipal allaient permettre aux aspirations locales de s'exprimer de façon plus modérée et moins partisane. Jusqu'à la fin de la décennie, et à l'apparition du mouvement annexionniste en 1849, aucune opposition articulée ne se manifesta; de même que le climat politique, la vie journalistique, dans le District de Saint-François, fut de ce fait beaucoup plus sereine dans les années quarante que dans les années trente.

\section{Conclusion}

Il ressort de ces analyses des premiers journaux du District de Saint-François quelques considérations qui mériteraient certainement de plus amples études:

77 Montreal Gazette (5 janvier 1839). La transaction de 1837 entre Armour et Walton ne faisait de celui-là entier propriétaire de l'entreprise qu'au paiement global des $£ 225$., échelonné sur douze mois (AJF, notaire Bureau: 867). Un seul numéro du Journal semble avoir été conservé (31 août 1839). Voir fiche signalétique no 7 .

78 Le numéro du Sherbrooke Gazette du 10 novembre 1849 porte les chiffres de série: VII $43 /$ Whole 565 . Si on se reporte 565 semaines avant cette date, on retrouve le $1^{\text {er }}$ janvier 1839, ce qui confirme la continuité de l'entreprise de Walton du

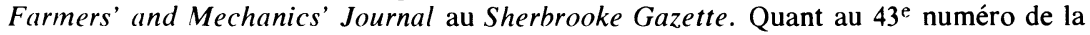
$7^{\mathrm{e}}$ année, il fait débuter la série à la mi-janvier 1843. Le journal porte encore son ancien titre en 1840 , puisque c'est sous ce nom que le service des Postes l'a transporté (Journaux de l'Assemblée législative, 1846, append. F, no 38) et en 1841, car il est cité ainsi par le Montreal Gazette (30 mars 1841).

79 Voir Paul G. Cornell, The alignment of political Groups in Canada 18411867 (Univ. of Toronto Press, 1962, 119 p.), 93-99. 
1. La presse des années 1830-1840 dans les comtés de Sherbrooke et de Stanstead est beaucoup plus riche, plus complexe et plus politisée qu'on ne le croyait: sept publications, aux couleurs partisanes très marquées font leur apparition dans la région et cristallisent les opinions contradictoires des populations.

2. Le phénomène de presse révèle par le fait même le tissu économique, social et politique des Cantons de l'Est durant les années qui précèdent la crise de 1837-38. Si la venue de la British American Land Company dans les Townships a été souvent retenue comme un des facteurs de radicalisation de l'aile papineauiste de l'Assemblée législative, les sentiments contradictoires qu'elle suscita dans la région ont été peu analysés. La lutte violente que se livrent en 1834 les deux journaux de Sherbrooke sur cette question illustre le clivage à la fois économique et politique de deux parties de la population: les tories, dont les chefs de file sont les marchands, contrôlent leurs employés, leurs apprentis, leurs sous-contracteurs et surtout leurs très nombreux débiteurs; les réformistes trouvent leurs partisans chez les cultivateurs aisés et non-endettés ${ }^{\circ 0}$.

3. Durant les années 1834-1838, l'acuité des tensions politiques au Bas-Canada a ses répercussions dans la presse des Cantons de l'Est: les journaux de la région sont plus nombreux, plus polémiques, plus vulnérables aussi à la concurrence et aux pressions de tout genre; aussi, se tournent-ils quelquefois vers les milieux journalistiques et politiques de Montréal; les cas du St. Francis Courier, renfloué par O'Callaghan, et du Sherbrooke Gazette, à l'époque de Robert Armour, illustrent bien ce lien entre la presse de Sherbrooke et celle de Montréal.

4. Néanmoins, on peut considérer cette influence montréalaise comme un phénomène déterminé par une conjoncture politique bien précise. De façon générale, la période 1823-1845 est sans doute ce qu'on pourrait appeler la "phase américaine» de l'histoire de la presse dans les Cantons de l'Est: les hommes, le matériel, les fournitures, souvent les nouvelles, parfois même les idées proviennent de la république voisine ${ }^{81}$. Nouveau témoignage de la vitalité de ce «second flot américain», qui, plus que les loyalistes et avant les

80 Vindicator (3 juin 1834): 3.

81 En 1845 , le fondateur du Stanstead Journal, Lee Roy Robinson, sera encore un journaliste originaire du Vermont. 
colons des Îles britanniques, constitua, dans la première moitié du dix-neuvième siècle, «le levain de la pâte des Cantons de l'Est ${ }^{82}$.

\section{ANNEXE \\ FICHES SIGNALÉTIQUES DES JOURNAUX DU DISTRICT DE SAINT-FRANÇOIS (1823-1845)}

1. British Colonist and St. Francis Gazette: hebdomadaire, paraissant le jeudi ; 4 pages, 5 colonnes; du $1^{\text {er }}$ mai 1823 à juin 1834. Publié à Stanstead Plain par Silas Horton Dickerson. Abonnement: 12s. 6d. ou en nature. Réformiste à partir de 1831 .

2. St. Francis Courier and Sherbrooke Gazette, hebdomadaire, paraissant le mardi, puis (1834) le jeudi; 4 pages, 5 colonnes; d'août 1831 à décembre 1835. Publié à Sherbrooke par Calvin Wilson Tolford. Abonnement: 10s. en espèces ou 12s. $6 \mathrm{~d}$. en nature. Réformiste à partir de 1832.

3. Farmers' Advocate and Townships Gazette: hebdomadaire, paraissant le lundi ; 4 pages, 5 colonnes; du 23 juin 1834 à juillet 1837. Publié à Sherbrooke par Walton et Gaylord. Rédacteur: Joseph Soper Walton. Abonnement: 10s. en espèces. Paiement en nature accepté. Constitutionnel.

4. Skylark: bi-mensuel (?); 4 pages, 2 colonnes; de juin à août (?) 1837. Publié à Sherbrooke. Prix au numéro: 2 pence. Satirique.

5. The Canadian Patriot: feuille irrégulière. Janvier-février 1838. Publié à Derby Line (Vt.) Rédacteur: Hiram F. Blanchard. Réformiste.

6. Sherbrooke Gazette and Townships Advertiser: hebdomadaire, paraissant le jeudi ; 4 pages, 5 colonnes; du 23 septembre 1837 à mars (?) 1838. Publié à Sherbrooke par Robert Armour, jr. Abonnement: 10s. Constitutionnel.

7. Farmers' and Mechanics' Journal and St. Francis Gazette: hebdomadaire, paraissant le samedi ; 4 pages, 6 colonnes; dès janvier 1839. Publié à Sherbrooke par Joseph Soper Walton. Titre modifié en janvier 1843 en Sherbrooke Gazette and Eastern Townships Advertiser.

82 Raoul Blanchard, Le centre du Canada français, III (Montréal, 1947): 329-331. 